\title{
Preparation of a New Type of Fiber Adsorbent Attached with Silica Microparticles
}

\author{
Takao Tsuda, ${ }^{\dagger}$ Mahoro Hisanaga, Takanori Inagaki, Shinya Kitagawa, and Kanji Miyabe \\ Materials Science and Engineering, Nagoya Institute of Technology, Gokiso, Showa, Nagoya 466-8555, Japan
}

\begin{abstract}
A new type of fiber adsorbent attached with silica microparticles was prepared. The silica microparticles were formed by the polymerization of silica oligomers on glass fibers, which were woven into a glass filter. The surface of the silica microparticles was chemically modified by bonding $\mathrm{C}_{18}$-ligands. SEM images indicated that the diameter of the uniform and spherical silica microparticles fixed on glass fibers was on the order of micrometers. It was confirmed that the glass filter adsorbent was effective for the adsorptive removal of toluene of low concentrations.
\end{abstract}

(Received March 23, 2004; Accepted May 20, 2004)

\section{Introduction}

Recently, much attention is being denoted to the air pollution due to volatile organic compounds (VOCs) of low concentrations in the atmosphere and houses. Even if the concentration of VOCs is low, it is possible that the intake of VOCs in human beings during a long period will have a negative influence on health. The determination of VOCs in the gaseous phase is usually carried out by combining an appropriate sampling and/or pretreatment procedure, and an instrumental analysis method, such as GC and GC-MS. The preconcentration of the objective VOCs and the separation from matrices are required for an accurate determination because their concentration is quite low.

Solid phase extraction (SPE) and solid phase microextraction (SPME) have been widely used in many cases of trace analysis. ${ }^{1-4}$ Although different types of adsorbents, such as activated carbon, carbon graphite, and porous organic polymers, have been used, $\mathrm{C}_{18}$-silica gel particles are the most widely used for the concentration and separation of VOCs. In practice, sample gases or liquids are passed through cartridges or disks packed with the $\mathrm{C}_{18}$-silica gel particles having a diameter of about $30-60 \mu \mathrm{m}$. Because of the low concentration of the objective VOCs, a large amount of the sample gases or liquids must be treated to achieve a high concentration ratio. A highspeed treatment is preferable for a reduction in the analysis time. In practice, however, there are some difficulties in the treatment of high flow rates: for instance, a rise of the pressure, the formation of channeling in the packed bed, and a leak of the objective component from the cartridges or disks. On the other hand, the objective components adsorbed must be desorbed from the adsorbents in order to supply them to the following GC or GC-MS procedure. It is also important how the objective components are easily desorbed. When a large amount of solvent is needed for the complete desorption of the objective component adsorbed, the concentration ratio is reduced due to a dilution. The desorption rate decreases with increasing particle

† To whom correspondence should be addressed.

E-mail: cct67333@syd.odn.ne.jp size because of the limited mass-transfer rate in the intraparticle space. This problem could be overcome by using adsorbents of much smaller particle size. However, the utilization of small particles results in some practical problems, as described above.

Some new types of adsorbents for SPE and SPME have been developed to overcome these drawbacks (described earlier). A fiber-type adsorbent coated with polymers is one of the alternatives for this purpose. ${ }^{3,5}$ Different polymers are used to prepare polymer-coated fibers, such as polydimethylsiloxane (PDMS) and polyacrylate. ${ }^{3,5}$ The volume of the polymer coating depends on the film thickness. A thick film coating is preferable to adsorb a large amount of the solute. On the contrary, however, a thick coating causes an increase in the analysis time and a reduction in the mass-transfer rate and hence, the column efficiency. On the other hand, the thermal stability of the coating fibers is another issue. Chong et al..$^{5}$ coated a fused-silica fiber with a bonded sol-gel layer of PDMS. They reported that the sol-gel PDMS fibers could be used in higher temperature ranges, compared with conventional coated PDMS fibers, and that the sol-gel coating technique is effective for improving the thermal stability of the coating fibers.

Lee et al ${ }^{6,7}$ prepared a new type of fiber adsorbent for SPME. Porous silica-gel particles for reversed-phase liquid chromatography (RPLC), such as $\mathrm{C}_{18^{-}}$and $\mathrm{C}_{8}$-bonded phases, were fixed on stainless-steel fibers by using a high-temperature epoxy. The large specific surface area of porous silica-gel particles is preferable to increase the adsorption capacity. The thin layer of the chemically bonded phases also contributes to the enhancement of the mass-transfer rate. It is expected that the attachment of porous silica-gel particles for RPLC on fibers remarkably improves both the adsorption capacity and separation efficiency. The authors compared the adsorption capacity of the porous layer thus prepared with that of polymer coated SPME fibers. They also studied the effect of the alkyl chain length and the functionality of the bonded phases on the selectivity for the extraction of polyaromatic hydrocarbons from aqueous solutions.

The porous layer fiber proposed by Lee et al.$^{6,7}$ consists of three different materials: stainless-steel fibers, porous silica-gel particles for RPLC, and an epoxy resin. The chemical homogeneity of the adsorbent surface is very important to 
prevent any unnecessary side reactions, and for the stable pretreatment of objective components. We prepared a new type of silica fiber adsorbent, which was attached with silica microparticles. ${ }^{8}$ Silica microparticles were prepared by the polymerization of silica oligomers under alkaline conditions and fixed on glass filters, which were woven into a glass filter. The surface of the silica microparticles was chemically modified by bonding $\mathrm{C}_{18}$-phases. A glass filter attached with $\mathrm{C}_{18}$-ligands bonded silica microparticles was used for the adsorptive elimination of toluene of low concentrations. The shape of the silica microparticles was observed by scanning electron microscopy (SEM).

\section{Experimental}

\section{Materials and reagents}

A glass filter sheet was offered from Hokuetsu Paper Mills, Ltd. (Tokyo, Japan). The glass filter is prepared by combining glass fibers $(93 \%)$ using acrylic resin $(7 \%)$. The thickness and weight of the glass filter are $0.37 \mathrm{~mm}$ and $63.2 \mathrm{~g} \mathrm{~m}^{-2}$, respectively. The diameter of the glass fiber is $0.6 \mu \mathrm{m}$. Silica oligomer was offered from PIATEC Co., Ltd. (Suzuka, Japan). It is obtained as an intermediate from the preparation process of silica gels. The degree of polymerization of tetrahydroxysilane is $10-20$. The silica oligomer is dissolved in a methanol/water (99/1, v/v) mixture. Its concentration is $30 \mathrm{vol} \%$. These data and information were provided from the manufacturers. Octadecyltriethoxysilane was purchased from Shin-Etsu Chemical Co., Ltd. (Tokyo, Japan). Other chemicals, i.e., toluene, $p$-xylene, sodium hydroxide, ammonia water, methanol, and ethanol were purchased from Wako Pure Chemical Industries, Ltd. (Osaka, Japan). They were super grade reagents.

\section{Preparation of glass filter adsorbent}

After the glass filter $(1.5 \mathrm{~g})$ was submerged in $40 \mathrm{~mL}$ of aqueous solution of sodium hydroxide $(1 \mathrm{M})$ under ambienttemperature conditions for $1 \mathrm{~h}$, the solution of sodium hydroxide containing the glass filter was heated at $80^{\circ} \mathrm{C}$ for 30 min. A glass filter treated by sodium hydroxide was washed with distilled water and centrifuged at $2000 \mathrm{rpm}$ for $1 \mathrm{~min}$ to reduce the water content. Then, the glass filter was washed with ethanol and similarly centrifuged at $2000 \mathrm{rpm}$ for $1 \mathrm{~min}$.

A 6-mL volume of methanol was added into the original silica oligomer solution of the same volume. The glass filter treated by alikali was submerged in $12 \mathrm{~mL}$ of the diluted silica oligomer solution ( $15 \mathrm{vol} \%)$ for $1 \mathrm{~h}$ at room temperature. Then, the glass filter was contacted with $20 \mathrm{~mL}$ of aqueous solution of ammonia (10 vol\%) for promoting the polymerization of silica oligomers. After the glass filter was centrifuged at $1000 \mathrm{rpm}$ for $5 \mathrm{~s}$ to eliminate any excess of ammonia, it was allowed to stand overnight. The glass filter was then heated in an electric oven according to a temperature program $\left(\right.$ at $80^{\circ} \mathrm{C}$ for $16 \mathrm{~min}$, $4^{\circ} \mathrm{C} \mathrm{m^{-1 }}$ to $180^{\circ} \mathrm{C}$, and at $180^{\circ} \mathrm{C}$ for $16 \mathrm{~min}$ ).

Octadecyltriethoxysilane (ODES) was dissolved with $p$ xylene. After the glass filter was impregnated with $15 \mathrm{~mL}$ of a $20 \mathrm{vol} \%$ solution of ODES, it was heated at $130^{\circ} \mathrm{C}$ for $1 \mathrm{~h}$. The glass filter was washed with $20 \mathrm{~mL}$ of ethanol and then distilled water. It was dried under reduced pressure for $2 \mathrm{~h}$.

\section{Imaging of the glass filter adsorbent attached with silica microparticles}

A JSM-5600 scanning electron microscopy (JEOL Ltd., Tokyo, Japan) was used to obtain SEM images of the glass filter adsorbent and the surface of silica microparticles.

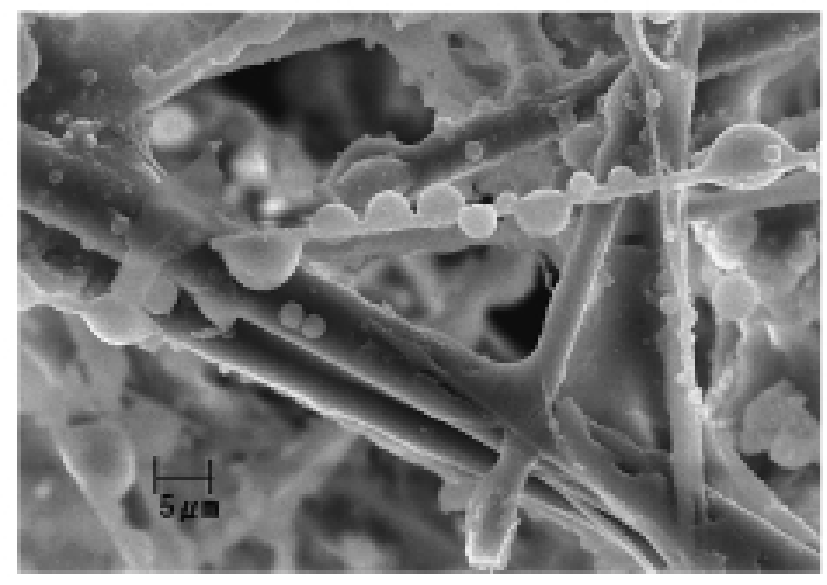

Fig. 1 Scanning electron micrograph of the glass-filter adsorbent at 1500-fold magnification.

\section{Flow experiments}

In this study, we chose toluene as a concrete component. Toluene is regarded as being one of the chemical materials, that are responsible for atmospheric pollution in a room (sick-house problem). The permissible limit of the concentration was established for toluene as $0.070 \mathrm{ppmv}$ by the Japanese government (the Ministry of Health, Labor, and Welfare). Adsorption experiments of toluene were carried out by means of the column method. The glass-filter adsorbent $(0.15 \mathrm{~g})$, which was attached with $\mathrm{C}_{18}$-ligand bonded silica microparticles, was packed into a polyethylene cylinder. The standard gas of toluene $(0.5 \mathrm{ppmv})$ was prepared by vaporizing liquid toluene in pure nitrogen and stored in a collapsible Tedlar $^{\circledR}$ bag, which was made of polyvinyl fluoride. Under ordinary temperature and atmospheric pressure conditions, $500 \mathrm{~mL}$ of the standard gas was passed through the packed bed of the glass-filter adsorbent while changing the flow velocity. The flow velocity of the feed gas was measured with a flow meter (TF-5310, YOKYO KEISO Co. Ltd., Tokyo, Japan). The treated gas leaving from the adsorbent bed was collected in another Tedlar ${ }^{\circledR}$ bag. A similar flow experiment was also made for the atmosphere in our laboratory.

\section{Analytical procedure}

The concentrations of toluene in the feed gas and the treated one were determined using a GC-FID (GC-17A, Shimadzu, Kyoto, Japan) equipped with a cold-trap system. A sample gas of $25 \mathrm{~mL}$ was passed through the cold-trap system at a flow rate of $50 \mathrm{~mL} \mathrm{~min}^{-1}$ for $30 \mathrm{~s}$. Then, the cold trap system was heated in order to inject the concentrated sample of toluene into the GC-FID system. A DB5-MS capillary column $(0.25 \mathrm{~mm}$ i.d. $\times$ $30 \mathrm{~m}$ length, film thickness $0.25 \mu \mathrm{m}$ ) and helium gas were used as the stationary and mobile phases, respectively. The temperature conditions of the GC-FID system ${ }^{9}$ were as follows: column oven $(323 \mathrm{~K})$, injector $(433 \mathrm{~K})$, and detector $(423 \mathrm{~K})$. The removal of toluene was calculated from the concentration of toluene at the inlet and outlet of the adsorbent bed.

\section{Results and Discussion}

\section{Structure of the glass filter adsorbent}

The SEM image of the glass filter adsorbent is illustrated in Fig. 1. Figure 1 demonstrates that a number of spherical 


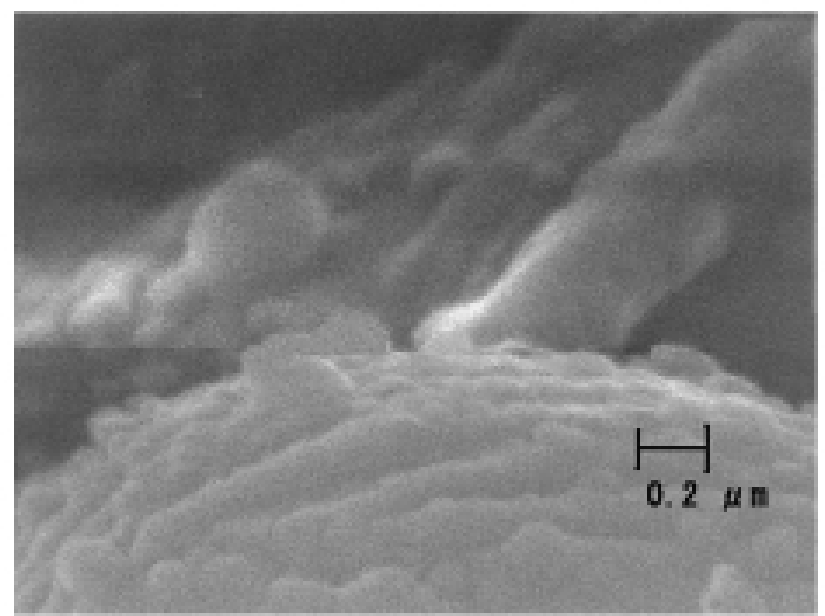

Fig. 2 Scanning electron micrograph of the surface of silica microparticles at 50000-fold magnification.

particles of silica attach to the surface of glass fibers. It is very important that the diameter of the silica particles is quite small, that is, on the order of micrometers, and that the silica microparticles have an almost homogeneous size distribution. In Fig. 1, however, it seems that the silica microparticles adhere to relatively thin glass fibers. Although there is no information about the influence of the size of the glass fiber on the formation and adhesion of the silica microparticles at this stage, further improvements are needed for preparing a suitable glassfilter adsorbent. Durability tests may also be required to clarify the chemical and mechanical stability of the glass filter adsorbent attached with the silica microparticles.

Figure 2 shows on enlargement of the surface of silica microparticles. An uneven surface is observed on the microparticles. It is indicated in Fig. 2 that the silica microparticles would heterogeneously grow at the active sites on the surface by the polymerization of silica oligomers. The attachment of a number of silica microparticles to the glass fibers and the unevenness of their surface contribute much to the increase in the surface area of the glass-fiber adsorbent, on which adsorption phenomena of the objective components take place. Additionally, the homogeneously small size of the silica microparticles must be preferable for the enhancement of the mass-transfer kinetics and the improvement of the column efficiency.

The chemical homogeneity of the glass-filter adsorbent developed in this study is another advantageous characteristic. In contrast with the porous layers previously developed for SPME, ${ }^{6,7}$ it basically consists of only silica-gel, and contains few impurities, such as stainless-steels (metals) and an epoxy resin (organic polymer). The chemically homogeneous surface is also very important for the materials used in SPE and SPME. The glass filter is applicable to a high-speed treatment of gaseous samples. Because glass fibers are woven into the glass filter with a large porosity, as illustrated in Fig. 1, it shows a low hydraulic resistance. As explained above, the glass-filter adsorbent attached with silica microparticles has many suitable characteristics for an adsorptive pretreatment and concentration of objective components.

\section{Calibration curve}

As explained earlier, toluene in both the feed and treated gases was determined by GC-FID equipped with a cold-trap system

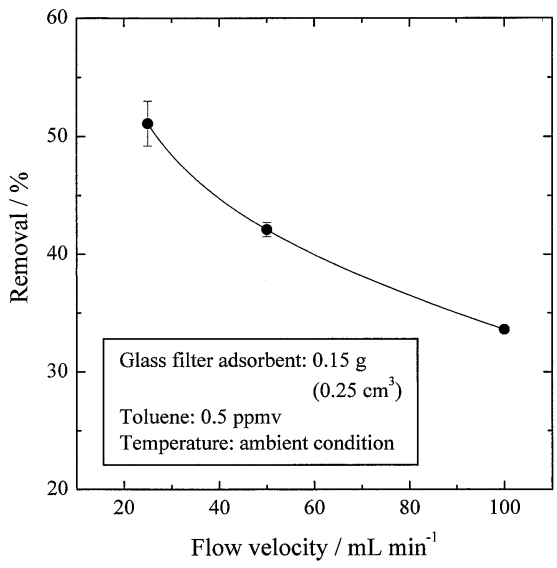

Fig. 3 Correlation between the removal of toluene and the flow velocity of feed gas containing toluene of 0.5 ppmv.

because the concentration of toluene was quite low in this study. At first, we confirmed the calibration curve for the gas chromatographic determination of toluene with the preconcentration using the cold trap system. A linear correlation was observed between the gas chromatographic peak area and the concentration of toluene in the range from 0.1 to 0.3 ppmv. The correlation coefficient was 0.97 . The relative standard deviation of the gas chromatographic determination of toluene was $3.9 \%(n=3)$ at the concentration of $0.1 \mathrm{ppmv}$.

\section{Adsorptive removal of toluene of low concentrations}

The surface of the silica microparticles was chemically modified by bonding $\mathrm{C}_{18}$-ligands. The hydrophobic surface would be applicable to the adsorptive removal of hydrophobic gases. As described earlier, toluene was selected as an objective component in this study. We tried to confirm the effectiveness of the glass-filter adsorbent for the adsorptive elimination of toluene of quite low concentrations.

Figure 3 shows the dependence of the removal of toluene on the flow velocity of the feed sample gas passing through the packed bed of the glass-filter adsorbent. The removal of toluene increases with decreasing flow velocity. When the flow velocity ranged from 25 to $100 \mathrm{~mL} \mathrm{~min}^{-1}$ (space velocity 6100 $24500 \mathrm{~h}^{-1}$ ), about $34-43 \%$ of toluene was eliminated from the feed gas containing toluene of $0.5 \mathrm{ppmv}$. The range of the flow velocity is relatively large compared with the ordinary process of gaseous adsorption. The range of space velocity in column operations using fixed beds is usually on the order of $10^{3} \mathrm{~h}^{-1}$ for gas phase adsorption and catalytic reactions.

Based on the results in Fig. 3, toluene included in the atmosphere of our laboratory was treated by the same column operation at a flow velocity of $25 \mathrm{~mL} \mathrm{~min}^{-1}$. The concentration of toluene was $0.13 \mathrm{ppmv}$ in the laboratory atmosphere. The concentration of toluene in the treated gas was determined to be approximately $0.07 \mathrm{ppmv}$. This is comparable to the permissible level of toluene described earlier. The removal of toluene is almost the same as the result of the above experiment using the standard gas. Because of the quite low concentration of toluene, the adsorption of toluene should take place under the conditions of a linear adsorption isotherm. In spite of the high flow velocity and extremely low concentration of toluene in the feed gas, about half of the toluene was removed. It is suggested that the glass filter adsorbent would be useful for the adsorptive elimination of toluene, as well as for the pretreatment procedure for analytical purposes. In the future, additionally, it should be 
confirmed that toluene can be quantitatively eliminated under low flow-rate conditions.

\section{Conclusion}

We prepared a glass-filter adsorbent attached with $\mathrm{C}_{18}$-ligands bonded silica microparticles. It has intrinsic structural characteristics, that is, a uniform and sufficiently small diameter, a spherical shape, and an uneven surface. The glassfilter adsorbent is basically chemically homogeneous. It mainly consists of silica and contains few impurities, such as metals and organic polymers. We also confirmed that the glass-filter adsorbent was effective for the elimination of toluene in quite low concentrations. The glass-filter adsorbent has some suitable and very important characteristics, which are required for materials used in the pretreatment procedure for analytical purposes. It is expected that the capabilities of this type of materials would be greatly advanced by further improvements.

\section{Acknowledgements}

The authors thank Hokuetsu Paper Mills, Ltd. and PIATEC Co.,
Ltd. for the generous offer of the glass filter and the solution of silica oligomer, respectively.

\section{References}

1. Z. Zhang, M. Yang, and J. Pawliszyn, Anal. Chem., 1994, 66, 844A.

2. J. S. Fritz and M. Macka, J. Chromatogr. A, 2000, 902, 137.

3. L. J. Krutz, S. A. Senseman, and A. S. Sciumbato, J. Chromatogr. A, 2003, 999, 103.

4. R. M. Smith, J. Chromatogr. A, 2003, 1000, 3.

5. S. L. Chong, D. Wang, J. D. Hayes, B. W. Whlhite, and A. Malik, Anal. Chem., 1997, 69, 3889

6. Y. Liu, Y. Shen, and M. L. Lee, Anal. Chem., 1997, 69, 190.

7. Y. Liu, M. L. Lee, K. J. Hageman, Y. Yang, and S. B. Hawthorne, Anal. Chem., 1997, 69, 5001.

8. T. Tsuda et al., US Patent, US 6, 2723, 157132.

9. K. Naitoh, Y. Inai, T. Hirabayashi, and T. Tsuda, Anal. Chem., 2000, 72, 2797. 